Check for updates

Cite this: J. Mater. Chem. B, 2020, 8, 1128

Received 16th October 2019 Accepted 9th January 2020

DOI: 10.1039/c9tb02289a

rsc.li/materials-b

\section{Dendrimer nanoparticles for colorectal cancer applications}

\author{
M. R. Carvalho, (D) abc R. L. Reis ${ }^{a b c}$ and J. M. Oliveira ${ }^{a b c}$
}

Cancer nanotechnology is a prolific field of research, where nanotools are employed to diagnose and treat cancer with unprecedented precision. Targeted drug delivery is fundamental for more efficient cancer treatments. For this, nanoparticles have been extensively used during the past few years in order to improve the specificity, selectivity and controlled release of drug delivery. It holds potential in minimizing systemic toxicity through the development of functionalized particles for targeted treatment. Among all the type of nanoparticles, dendrimers display several advantages, which make them ideal candidates for improved and targeted drug delivery in cancer research. Dendrimers can transport large amounts of drug into specific areas. In addition, they can be employed for monitoring the progress of the treatment process, with an unprecedented theranostic capability. Special emphasis is given to colorectal cancer and to the preferred employed strategies for producing drug-loaded/functionalized NPs for cancer therapy in the past few years.

\section{Introduction}

Nanotechnology is an emerging field of research that is expected to play a definitive role in biomedicine in the near future, particularly for the targeted delivery of drugs at pathological sites inside the human body. ${ }^{1}$ Nanoparticles (NPs) can be defined as particulate matter ranging between 1 and $100 \mathrm{~nm}$ in diameter, with a high surface area-to-volume ratio, and specialized surface characteristics. ${ }^{2-4}$ The use of NPs as drug carriers in oncology started in 1986, when it was reported that NPs showed a tendency to accumulate in tumoral tissues. ${ }^{5}$ This passive accumulation, also known as passive or primary targeting, is known as the "Enhanced Permeability and Retention" (EPR) effect and is one of the milestones of cancer treatment using NPs. Therefore, the use of NPs for cancer therapeutics is very promising due to their high specificity and accumulation in tumor sites as well as their long blood circulation time (Fig. 1).

However, one of the major problems related to cancer treatment is the intrinsic anti-cancer drug resistance, which can appear prior to chemotherapy, as well as acquired resistance due to drug treatment. ${ }^{6}$ The selectivity improvement yielded by

\footnotetext{
${ }^{a} 3 B$ 's Research Group, I3Bs - Research Institute on Biomaterials, Biodegradables and Biomimetics, University of Minho, Headquarters of the European Institute of Excellence on Tissue Engineering and Regenerative Medicine, AvePark, Parque de Ciência e Tecnologia, Zona Industrial da Gandra, 4805-017 Barco, Guimarães, Portugal.E-mail: miguel.oliveira@i3bs.uminho.pt

${ }^{b}$ ICVS/3B's - PT Government Associate Laboratory, Braga/Guimarães, Portugal

${ }^{c}$ The Discoveries Centre for Regenerative and Precision Medicine, Headquarters at University of Minho, Guimarães, Portugal
}

NPs resulted in a great enhancement in the efficacy of the transported drug, while the occurrence of side effects in the patient was reduced. Hence, targeted nanomedicine offers innovative therapeutic strategies to overcome the various limitations of conventional chemotherapy, such as drug resistance, enabling enhanced selectivity, and early and more precise cancer diagnosis. ${ }^{7}$ Moreover, it is possible to incorporate targeting moieties selective for cancer cell biomarkers, which improves even more the selectivity and specificity of the treatment. ${ }^{8}$ The main types of targeting moieties that are used for decorating and targeting NPs are: (i) antibodies; ${ }^{9}$ (ii) peptide-based targeting; ${ }^{10}$ (iii) small molecule-based targeting; ${ }^{11}$ and (iv) aptamer-based targeting. ${ }^{12,13}$

The discovery of non-biodegradable nanoparticles including micelles, nanogels, liposomes, nanoemulsions, polymeric NPs, gold NPs and magnetic NPs as agents in nano-drug delivery and

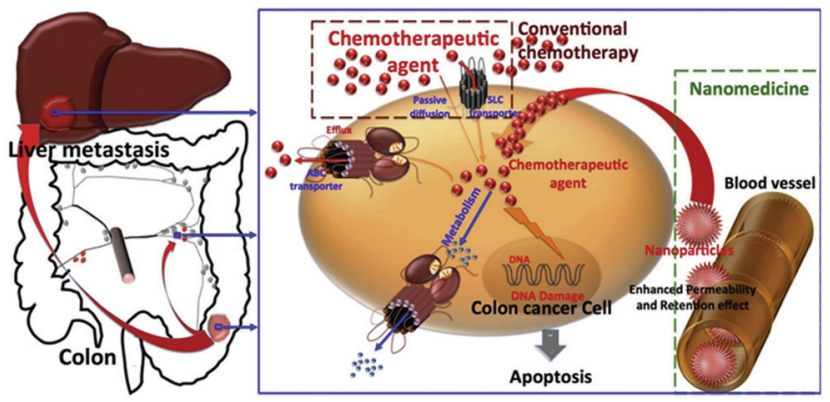

Fig. 1 Rationale for the nanomedicine approach in cancer-therapy. Reprinted with permission from ref. 1. 
Table 1 Current nanotechnology applications in colorectal cancer clinical trials and status

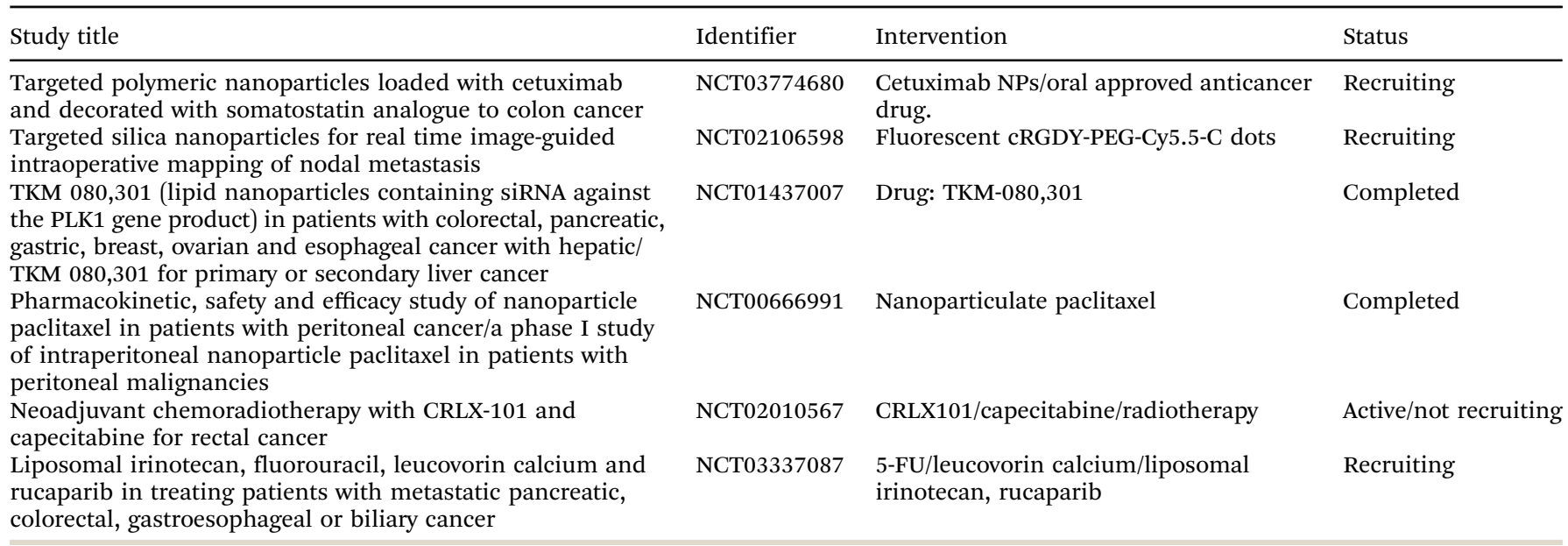

imaging at pathological sites has enhanced delivery at lower doses and increased the aqueous solubility and bioavailability of the drug with reduced side effects. ${ }^{14}$ Nevertheless, the clinical application of these NPs to CRC therapy remains limited, as can be seen from Table 1 .

Moreover, as can be seen from Table 1, none of the ongoing clinical trials using nanotechnology in colorectal cancer comprises the use of dendrimer nanoparticles. After literature research, it was found that there is only one clinical trial using dendrimers, with application in inoperable liver cancers. This dendrimer consists of poly-L-lysine dendrimer as a nanovector mixed with a complex of 188-rhenium-ligand (nitro-imidazole-methyl-1,2,3triazol-methyl-di-[2-pycolyl]amine). ${ }^{15}$ Although it is a very popular type of nanoparticle inclusively used in clinical trials for other pathologies such as breast cancer and HIV prevention (VivaGel $^{\circledR}$ ), scientific advances are needed for dendrimer applications in clinical settings.

A variety of dendrimers have been developed and used since the 1980s, but the ones derived from polyamidoamine (PAMAM) are undeniably the most employed. They are generally biocompatible and non-immunogenic systems, which favors their use in drug delivery. The core of PAMAM is most commonly ethylenediamine, although more hydrophobic molecules such as diaminododecane, diaminohexane, and diaminobutane may also be used. ${ }^{16}$

Recent literature suggests that dendrimers will be a solid bet in the future of cancer therapeutics. ${ }^{17}$ This is because the field of oncology could be transformed by novel diagnostic and therapeutic strategies based on dendrimer nanoparticles. This advanced nanotheranostics area may include improved imaging techniques (such as MRI) by using dendrimers as new contrast agents. Dendrimers will also be applied for targeting a diverse variety of cancers, advancing on their safety and efficacy. In this regard, one of the most interesting targeting strategies involves anti-metabolite drugs, vitamins or hormones that tumors need for growth. Finally, further cancer-related applications of dendrimers in areas such as photodynamic therapy, boron neutron capture therapy, and gene therapy are being studied. ${ }^{18}$ This review will cover the fundamentals of research utilizing dendrimers for cancer diagnosis and therapy, with a special focus on colorectal cancer (CRC).

\section{Dendrimer nanoparticles}

Dendrimers are in the category of polymer nanoparticles. However, they have a very different structure from classical polymers, which makes them unique. They consist of globular molecules made out of branched layers (generations). Such a precise synthesis leads to obtaining monodisperse molecules. On their outer surface, dendrimers can be engineered to have various functional groups such as $\mathrm{COOH}, \mathrm{COONa}, \mathrm{NH}_{2}$, or $\mathrm{OH} .{ }^{19}$ Therefore, after very simple surface modification, dendrimers render intelligent nanoparticles, transporting drugs into specific areas and at the same time can be used for monitoring the state of organs attacked by cancer cells, as well as the progress of the curing process. They can help to limit the anti-cancer drug delivery to designed goals only, eliminating many side effects of chemotherapy. ${ }^{20}$ Adding to this, to visualize the effectiveness of targeting, other moieties such as imaging agents can be attached to dendrimers and then assessed by MRI or CT. Because of this chemical multifunctional therapeutic delivery, dendrimer-based nanoparticles have received considerable attention in cancer research. ${ }^{21}$

\subsection{Properties}

Among the innumerous types of NPs available, dendrimers offer multiple advantages. They are highly branched polymers, and therefore specific moieties and drugs can be easily conjugated and encapsulated. PAMAM dendrimers are the most common class of dendrimers, suitable for many tissue engineering and regenerative medicine (TERM), materials science, and biotechnology applications. ${ }^{22}$ They contain an inner alkyl-diamine core and a peripheral shell made of tertiary amine branches. ${ }^{23,24}$ Therefore, the high level of control over dendritic architectures makes dendrimers ideal carriers in biomedical applications. ${ }^{25}$ In addition, the toxicity of dendrimers mainly comes from the high cationic charge density in the periphery, where charges interact 


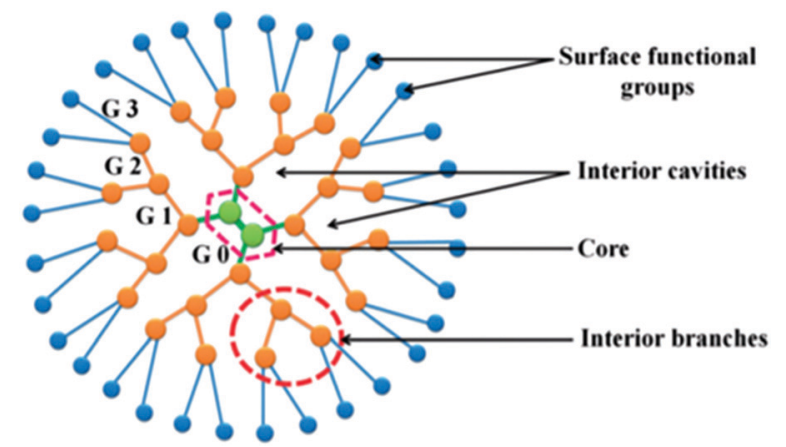

Fig. 2 Dendrimer branched architecture, representing the increasing generations (G). Adapted from ref. 24.

with the biological cell membrane, resulting in membrane disruption, a hurdle that can be easily overcome by surface modification. Dendrimers have the advantages of being biocompatible upon modification and can be easily eliminated from the body ${ }^{26}$ through the kidneys along the same metabolic pathways taken by folate, peptides and antibodies. ${ }^{27-30}$ The impact of size on the in vivo behavior of dendrimers is well known. In general, low generation dendrimers (e.g., G5 or smaller, with hydrodynamic radii of $<3.5 \mathrm{~nm}$ ) display relatively rapid clearance from blood and facile elimination into urine. On the other hand, PEGylated dendrimers or higher generation dendrimers (G6 and above) tend to circulate longer and evenly distribute within the major peripheral organs. In addition, only trace amounts were reported to be transported across an intact blood-brain barrier. ${ }^{31}$ In addition to urinary excretion, there is also some evidence to suggest that excretion via the feces may play a significant part in dendrimer elimination and that in some cases this is dependent upon dendrimer generation..$^{30}$ Limited mechanistic studies for renal retention of PAMAM dendrimers report the localization of these polymers in the lysosomes of proximal tubule cells. ${ }^{32}$

In a deeper study, the biodistribution of PAMAM labeled D-Cy5 was studied by injection in healthy rabbits on day 5 of life with the highest accumulation in the bladder and kidneys, confirming its fast renal clearance, even at an earlier age. ${ }^{31}$ The present study suggests that more than $90 \%$ of the injected dose of the G4 dendrimer is cleared out from the newborn rabbits over $24 \mathrm{~h}$, with less than $5 \%$ in blood circulation. ${ }^{31}$

Since dendrimers possess high density of surface functional molecules, they are easily conjugated with several targeting agents for selective delivery of chemotherapeutics to the tumor tissue. Furthermore, they contain internal cavities for macromolecule encapsulation, enabling them to transport highly hydrophobic drugs (Fig. 2). ${ }^{33}$

\subsection{Synthesis and types of dendrimers}

Dendrimers have been traditionally synthesized by two major routes: the divergent method, introduced by Tomalia et al. ${ }^{24}$ and the convergent one, developed by Hawker and Frechet. ${ }^{34}$ In the first method (divergent), the final molecule grows radially from a core by the sequential addition of layers of monomers, with each layer constituting a new generation (G). The number of surface groups multiplies according to the functionalities in each monomer ramification. ${ }^{35}$ It is important that every step of the reaction is fully completed before the addition of a new generation to avoid defects in the branches. One of the main advantages of this approach is that in the final step of the reaction, the surface of the dendrimer can be easily modified with desired functional groups. Moreover, it is a fast synthesis which allows the preparation of large dendrimers. The main drawbacks of this approach are that extensive purification is required, since the final product and the intermediate reactants have similar molecular weights, charge, and polarity. ${ }^{36}$ The main methods of purification used in this type of synthesis are dialysis and precipitation of dendrimers. ${ }^{37}$ Also, the higher the generation, the greater the chances are of having branching defects, since the presence of bulky branches creates difficulties in the coupling of new ones. Despite these obstacles, the advantages of this strategy have made it the main route for dendrimer production.

In a reverse way from the divergent synthesis, dendrimers can also be synthesized starting from the surface towards the inner core. The growth of the molecule starts from the ends of the chain, beginning by integrating the various branching points with other monomers that will constitute the dendrimers. Finally, these branches are attached to a central core when they reach the desired generation size. ${ }^{38}$ In contrast to the divergent growth, this method permits easier purification due to bigger differences between the final products and the initial reagents. The number of reaction steps required for dendrimer synthesis and purification in the convergent method can be reduced, making the preparation of higher generation dendrimers more efficient compared to the divergent method. ${ }^{30,39}$ Also, impurities generated during convergent synthesis can be easily separated as these impurities are usually very different from the synthesized dendrimers in size, structure, and physical properties.

Other advantages include higher monodispersity for low generations and fewer branch defects. The main downsides are low yield and difficulties in obtaining higher generations due to steric interferences when the branches are connected to the core. ${ }^{40}$

Regarding the available types of dendrimers, poly(propylene imine) (PPI) dendrimers were the first ones to be reported in the 70's. Together with PAMAM, they represent the most widely studied type. PPI dendrimers can be based on a 1,4-diaminobutane (DAB) core, but can also be synthesized from an ethylenediamine nucleus and other core molecules by a double Michael addition reaction. Hence, their interior contains various tertiary tris-propylene amines, and they form full generations with primary amines as the surface ends. ${ }^{41}$

Another common type of dendrimers is the poly-L-lysine (PLL) dendrimers, mostly used as gene carriers due to their excellent condensation with oligonucleotides. Among their favorable characteristics are good biocompatibility, water solubility, biodegradability, and flexibility, similar to other dendrimers. With peptide bonds in their structures, both their core and branching units are commonly based on the amino acid lysine, so PLL dendrimers differ from the general concept of 


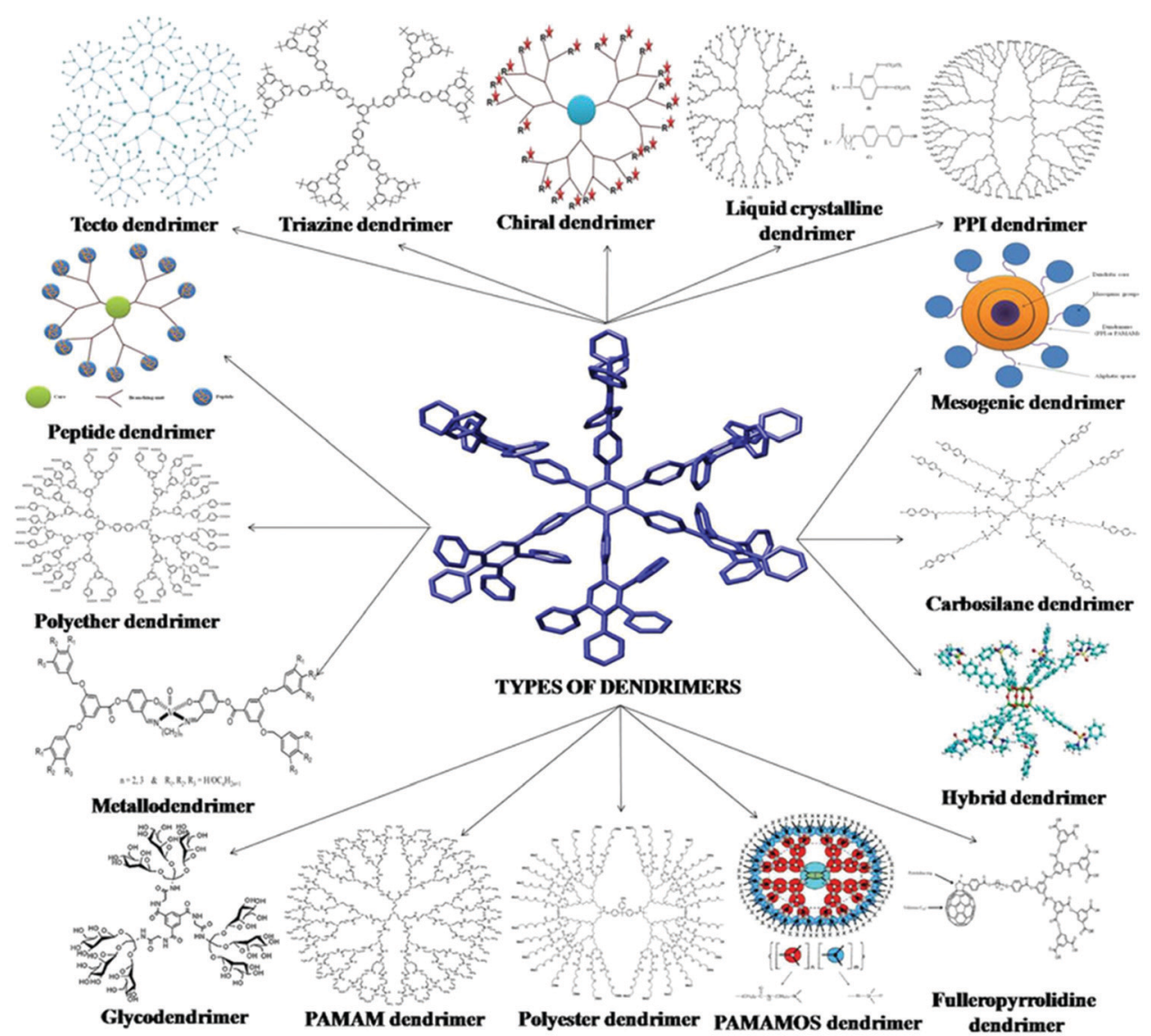

Fig. 3 Different types of dendrimers. Reprinted with permission from ref. 44.

PAMAM and PPI dendrimers as they are mostly asymmetrical. ${ }^{42}$ However, they are still precise molecules, with a controlled number of lysines branching out from the core and terminal amine residues. The lysine in the terminal group of PLL contains two primary amines that are frequently modified for better biological performance. ${ }^{21,43}$

There are many more types of dendrimer nanoparticles, although less used. They can be classified according to their shape, structure, branching, solubility, chirality and attachment, which can be seen from Fig. 3 .

When compared to other dendritic NPs, PAMAM surpasses them due to the ease of its preparation, desirable chemical and physical properties, surface functional groups, and comparatively lower toxicity to other dendrimers. ${ }^{45,46}$

The physical and chemical features of dendrimers, which include high monodispersity, water solubility, encapsulation ability, and large number of functionalizable peripheral groups, make these macromolecules suitable contenders as drug delivery vehicles for cancer therapeutics. ${ }^{39}$ Moreover, dendrimers are prepared with a level of control unattainable with most linear polymers, leading to nearly monodisperse, globular macromolecules with a large number of peripheral groups. ${ }^{47}$

PAMAM dendrimers can be easily prepared using the iterative reaction sequence developed by Tomalia et $a .^{48}$ and are easily conjugated with various functionalities. The novelty was obtaining these macromolecules by repeating a chemical procedure as follows: the most applied divergent synthesis of PAMAM dendrimers begins with an amine functional core unit that is reacted with methylacrylate by the Michael addition chemical reaction. This results in the synthesis of two new branches per amine group with an ester-terminated dendrimer, which is known as a "half-generation" dendrimer. ${ }^{49}$ Further amidation of the methyl ester with ethylene diamine results in "full-generation" amine-terminated dendrimers. Reoccurrences of Michael addition and amidation steps yield the next-higher generation dendrimer with additions to its molecular weight, number of terminal functional groups, and size. ${ }^{46}$

Functionalization is an integral part of dendrimers' multiple uses. Functionalization is the process of incorporating multiple active sites in dendrimers in order to generate macromolecules with multifunctional architecture. ${ }^{50,51}$ Functionalized dendrimers can have six distinct features known as "Critical Nanoscale Design Parameters" (CNDPs). These parameters consist of size, shape, surface chemistry, flexibility, rigidity, architecture and elemental composition. ${ }^{34}$ Depending on the clinical application of the dendrimer nanoparticles, these features can be changed and studied, changing the intrinsic properties, function and performance of the nanoparticles. Surface functionalization of dendrimer nanoparticles usually renders increased specificity of the dendrimer to improve the efficiency of cancer therapy and also 
an increase in its circulation time. ${ }^{52}$ For instance, dendrimer nanoparticles find useful functionalization in the following areas: to (i) increase biocompatibility (by PEGylating ${ }^{53}$ or acetylation $^{54}$ ); (ii) enhance transfection efficiency (usually by amino $\operatorname{acid}^{55}$ or lipid functionalization $^{18}$ ); (iii) induce site specific delivery (e.g. aptamer, ${ }^{56}$ antibody, ${ }^{57}$ vitamin, ${ }^{58}$ peptides ${ }^{59,60}$ ); or (iv) render stimuli responsive dendrimer nanoparticles (e.g. $\mathrm{pH},{ }^{61}$ thermo, ${ }^{62}$ photo, ${ }^{63}$ redoxresponsive $^{64}$ ).

When it comes to dendrimer targeting, aptamers are comparable to monoclonal antibodies, being well-established therapeutic molecules in terms of specificity and affinity to the target. Aptamers are small enough to enter deep into tumors, showing limited immunogenic effects. Because of these advantages, the use of aptamers is a promising strategy to overcome existing problems of common anticancer therapies. But the advantage of aptamers over antibodies includes their high stability, ease of synthesis, less batch-to-batch variation and facile chemical modifications that allow different conjugation chemistries. ${ }^{56,65}$ Regarding peptide strategies, there are numerous tumor-specific targets, and the peptides that bind them are usually divided into three categories: (a) peptides that bind to cell surface receptors; (b) peptides that target intracellular receptors; and (c) peptides that specifically interact with the extracellular matrix. When comparing peptides to antibodies, the latter presents inherent deficits that may limit their in vivo applications. Their large molecular weight results in slow delivery and diffusion into tumor tissues. Another drawback includes the limited stability of antibodies; their activities depend on their intact spatial conformation, which is problematic for in vivo drug delivery systems and causes storage and transportation problems. ${ }^{60}$

Efforts can also be made namely to reduce cytotoxicity and enhance transepithelial transport, ${ }^{37}$ for interaction with coupling molecules such as natural-based polymers, ${ }^{37}$ fluorescent probes, ${ }^{37}$ and an inner hydrophobic core where other molecules can be trapped. ${ }^{66,67}$ Among the myriad possibilities, dendrimers can also find applications as imaging agents ${ }^{66,68,69}$ and scaffolds for TERM. ${ }^{70}$

\section{Dendrimer applications in cancer research}

A strong body of evidence now suggests that NPs in the form of dendrimers may be of added value in the future of oncologyrelated theranostics. ${ }^{71-73}$ Other than the flexibility for functionalization using diverse ligands and its low-nanometer size, the introduction of stimuli responsive functionalities on dendrimers allows the release of payloads in response to specific triggers, as discussed before. These triggers could be endogenous in nature (acid, enzyme, and redox potentials) or it could be applied externally (light and temperature). ${ }^{74-76}$ One of these examples is the recent work developed by Nigam et al. ${ }^{77}$ on cervical cancer, where iron oxide NPs were modified with Generation 2 (G2) PAMAM dendrimers and loaded with doxorubicin (DOX), therefore combining magnetic chemotherapy and hyperthermia on HeLa cancer cells. ${ }^{77}$ When exposed to an alternating current magnetic field, the results show enhanced cell death as a result of the fatal synergistic contribution of DOX and high temperatures. Interestingly, the combinatorial treatment reduced the cancer cell viability from $100 \%$ to $3.6 \% .^{77}$

For targeting purposes, folic acid was conjugated to PAMAM dendrimer NPs. ${ }^{78}$ The results showed negligible toxicity towards non-cancerous MRC9 lung fibroblast cells, as well as the ability of dendrimer nanoparticles for targeted co-delivery of siRNA and chemotherapy agents together in the lung cancer cells. ${ }^{78}$

For breast cancer, one good example of a dendrimer application is the work of Chittasupho et al. ${ }^{79}$ Bearing in mind that breast tumors preferentially metastasize to the lung, bone and distant lymph nodes (the secret high levels of CXCL12), the team hypothesized that targeted inhibition of CXCR4 in breast cancer cells should suppress CXCR4-positive tumor cells toward secondary metastatic sites. The results showed enhanced in vitro cellular toxicity as compared with non-targeted dendrimers. The modified dendrimers exhibited remarkably reduced migration of BT-549-Luc breast cancer cells towards the used chemoattractant. This report demonstrated the potential utility of LFC131dendrimer conjugates for breast cancer therapy and metastasis.

In the case of liver cancer, due to lack of cell surface biomarkers and highly metastatic nature, early detection and targeted therapy of hepatocellular cellular carcinoma (HCC) is an unmet clinical need. ${ }^{80}$ Galactosamine (Gal) is among the few selective ligands used for targeting HCCs due to its high binding affinity to asialoglycoprotein receptors (ASGPRs) overexpressed in HCC. In a recent work, Yousef et al. ${ }^{80}$ engineered a nanoscale G4 PAMAM dendrimer NP anchored to Gal and loaded with the potent anticancer curcumin derivative (CDF) as a platform for targeted drug delivery to HCC. Surprisingly, in an in vivo xenograft model, cytotoxicity assays in HCC cell lines showed that CDF was more potent as a chemotherapeutic anticancer agent than the currently in use Doxorubicin, Sorafenib and Cisplatin chemotherapeutic agents. ${ }^{80}$

Glioblastoma is the most common type of malignant brain tumor and one of the deadliest cancers. ${ }^{81}$ It has been described that dendrimers have affinity to cross the blood-brain barrier after systemic administration. ${ }^{82}$ Liu et al. ${ }^{83}$ utilized a combined chemo- and gene-therapy approach for effective glioma treatment and developed a DOX-loaded dendrigraft poly-L-lysine (DGL) dendrimer surface modified with TNF (tumor necrosis factor) related apoptosis-inducing ligand (TRAIL) for the tumor targeting specifically of T7 peptide, a TfR-specific peptide. This approach was based on the knowledge that DOX increased the anticancer effect of TRAIL by regulating the expression of death receptors, as well as the stimulation of apoptotic pathways. ${ }^{83,84}$

For example, Langereis et $a l^{85}$ reported the synthesis of 5-6 nm gadolinium-diethylene triamine penta-acetic acid (Gd-DTPA)-terminated poly(propylene imine) (PPI) dendrimers as an MRI agent. Talanov et al. ${ }^{86}$ reported a PAMAM dendrimerbased nanoprobe with dual MR and fluorescence (Fl) modalities. Gd(III) was covalently attached to a dendrimer to create a fresh macromolecular contrast MRI agent. The authors used 2-(4-isothiocyanatobenzyl)-6-methyl diethylenetriaminepentaacetic acid (1B4M-DTPA) and Cy5.5 as a bifunctional chelating agent. 
The PAMAM dendrimers covalently attached to the Gd(III)-DTPA chelates and units of the near-infrared (NIR) fluorescent dye, Cy5.5, to form a dual-modality MRI-FI agent. ${ }^{86}$

They can be easily functionalized with a diverse variety of ligands to reach the tumor through the different body barriers in the body with minimal loss of activity. This results in the selective targeting and killing of tumor cells without affecting the normal cells and most importantly, with an actively controlled release mechanism.

\subsection{Dendrimer nanoparticles in CRC}

CRC is the third most diagnosed cancer in the world. It manifests as a malignant neoplasm in the mucosa of the colon or the rectum. ${ }^{87}$ Despite notable progress in treatment, CRC still leads to significant morbidity and mortality. ${ }^{88}$ Colorectal cancer is a heterogeneous group of diseases. These have individual genetic and epigenetic backgrounds. In order to improve clinical management and better predict patient outcome, classification of colorectal cancers based on location, histology, etiologic factors, and molecular mechanisms of tumorigenesis has been performed. ${ }^{89}$ Their effectiveness of chemotherapy remains limited due to the intrinsic build-up of resistance of cancer cells to chemotherapy drugs, dose-limiting toxicities and other major side effects. New strategies to overcome these issues are being developed, one of which is cancer nanomedicine, a rapidly developing interdisciplinary research field. ${ }^{90}$

The chemotherapeutic approach alone has not been found to be very efficient in CRC as the drug molecules may not reach the target site with an effective concentration and suffer a nonspecific distribution, with only a small fraction of the drug reaching the tumor. ${ }^{91,92}$

These unique "polymeric compounds" can form intelligent species after modification, transporting drugs into specific areas and at the same time can be used for monitoring the state of organs attacked by cancer cells, as well as the progress of the curing process. ${ }^{14}$ Recent advances in nanotechnology have rendered it an attractive approach for designing novel clinical solutions for CRC. ${ }^{14}$

When tumor cells are shed from primary tumors or metastatic sites of early-stage cancer patients and enter the bloodstream, they are called circulating tumor cells (CTCs). ${ }^{93}$ CTC-driven cancer relapse and metastasis are the leading causes of cancer-related death worldwide. Owing to the importance of CTCs as an indicator of poor prognosis, various approaches were exploited to efficiently isolate and capture CTCs from large populations of interfering cells. Although several technologies (such as microfluidics-based, size-based filtration, etc.) have been developed, they were not truly

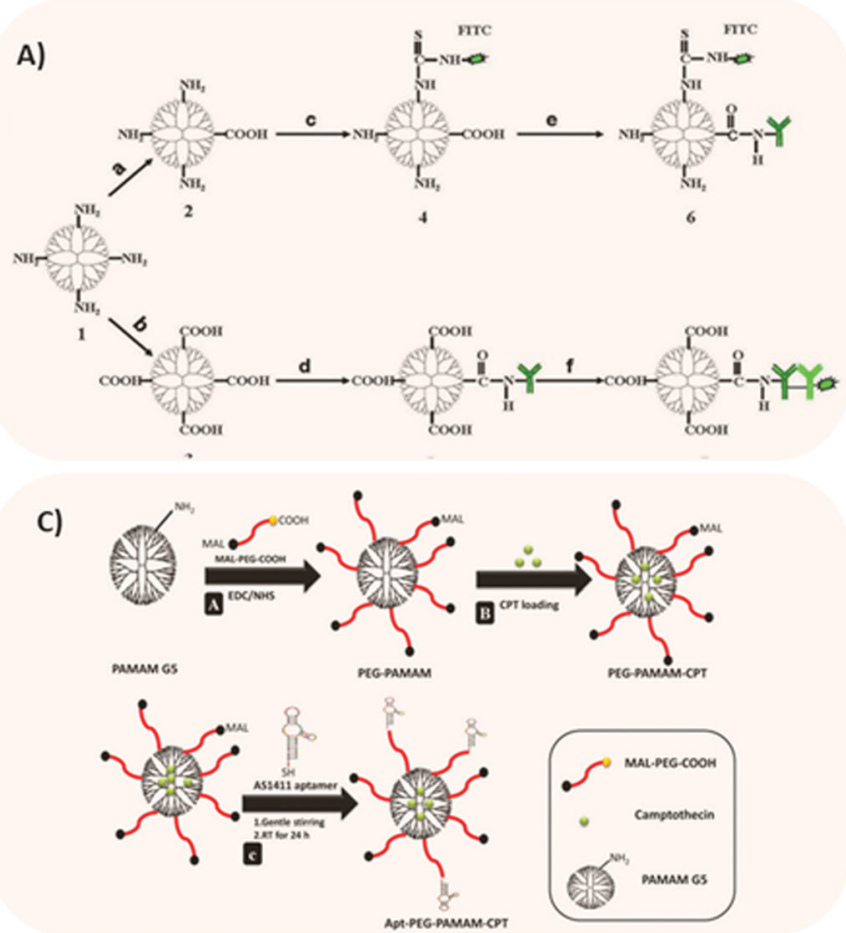

B)

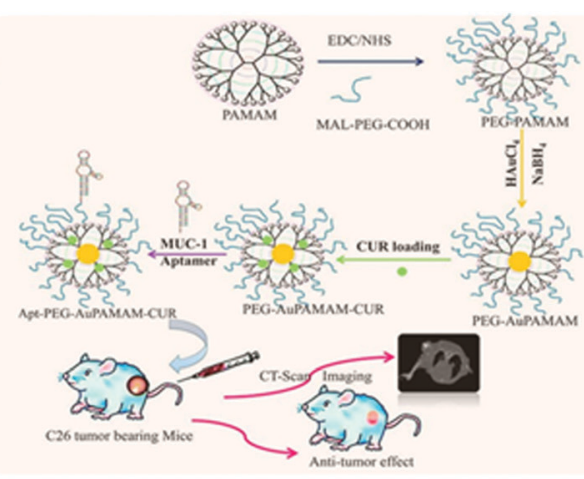

D)

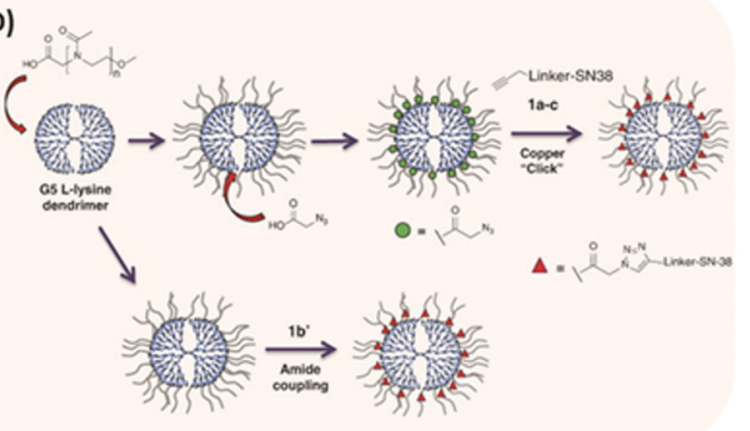

Fig. 4 Different strategies for employing dendrimer NPs in CRC research. (A) Two synthetic procedures of aSlex-conjugated dendrimers with FITC labeling. Reprinted with permission from ref. 93. Copyright (C) 2015 Nature. (B) Dendrimer-gold hybrid structure synthesized by complexing AuCl${ }_{4}^{-}$ions $^{-}$ with PEGylated amine-terminated generation 5 PAMAM dendrimer. The resultant hybrid system was loaded with curcumin. The curcumin-loaded PEGylated Au dendrimer was further conjugated and tested, in vitro and in vivo, to MUC-1 aptamer for targeting colorectal adenocarcinoma. Reprinted with permission from ref. 12. Copyright (C) 2018 Elsevier. (C) Schematic representation of (A) synthesis of pegylated PAMAM dendrimer (PEG-PAMAM); (B) camptothecin (CPT) loading in the cavities of PEG-PAMAM; (C) conjugation of thiolated AS1411 aptamers to the maleimide groups of MAL-PEGPAMAMCPT and preparation of Apt-PEG-PAMAM-CPT. Reprinted with permission from ref. 95. Copyright (C) 2017 Elsevier. (D) Synthesis of polyoxazoline-modified dendrimers. Reprinted with permission from ref. 97. Copyright (C) 2017 Elsevier. 
successful. So, a team developed a nanotechnology-based cell detection and capture method. ${ }^{93}$ Purified anti-Slex (aSlex) antibody and FITC labeled-second antibody (IgG/IgM-FITC) were used for the following synthesis of aSlex-coated dendrimer conjugates. By doing this, dendrimers would capture (attach to) saliva acidifying Louis oligosaccharides X (Sialyl Lewis X, Slex, a type II carbohydrate antigen for mediating the colorectal cancer metastatic process). The colorectal CTCs were not only captured in artificial blood samples but restrained in cell activity by the conjugates, which means, according to the referred paper, that there was a specific interaction between antigen (present in CTCs) and antibody (present in dendrimers). Xie et al. ${ }^{93}$ reported an effective approach to specifically bind and capture colon cancer HT29 cells by using multiple Sialyl Lewis X antibodies (aSlex)-conjugated with PAMAM dendrimers (Fig. 4A). The results indicated that the conjugate showed the enhanced capture of HT29 cells in a concentration-dependent manner and the maximum capture efficiency of $77.88 \%$ was obtained within 1 hour exposure. ${ }^{93}$ This work provided novel conceptual guidance for the effective prevention of cancer metastasis.

Capecitabine is one of the most used anticancer drugs in CRC and is converted into 5-FU by various metabolic enzymes involved in DNA damage and tumor growth inhibition. ${ }^{94}$ However, capecitabine has multiple adverse side effects affecting the blood, hair cells, bone marrow, and liver. In this context, Nabavizadeh et al. $^{92}$ induced colon adenocarcinoma in mouse models with azoxymethane, a carcinogen agent, and then investigated the potentiality of non-modified G4 PAMAM dendrimers to improve the capecitabine therapeutic index and decrease its adverse side effects on the liver and bone marrow. ${ }^{92}$ Although no targeting was performed, the team compared the effects of the free and conjugated capecitabine form on tumor size and blood cell line abnormalities. The results showed reduced side effects in the liver and blood along with decreased tumor size when compared with the free form. ${ }^{92}$

In a recent work, Alibolandi et al. ${ }^{12}$ encapsulated gold ( $\left.\mathrm{Au}\right)$ NPs inside PAMAM dendrimer NPs. The aim of this study was to investigate the theranostic capability of curcumin-loaded dendrimer-gold hybrid structures (Fig. 4B).

The obtained results confirmed higher cellular uptake, internalization and cytotoxicity of Apt-PEG-AuPAMAM-CUR in comparison with PEG-AuPAMAM-CUR in C26 and HT29 colorectal cancer cell lines. Moreover, the system worked as an effective anti-tumor therapy and accurate computed tomography imaging of C26 tumor-bearing mice due to gold accumulation. $^{12}$
Similarly, Alibolandi et al. ${ }^{95}$ developed camptothecin-loaded pegylated PAMAM dendrimer (Fig. 4C). For targeting purposes, the team functionalized the system with AS1411 anti-nucleolin aptamers for site-specific targeting against CRC cells, which overexpress nucleolin receptors. ${ }^{95}$ Remarkably, aptamer AS1411 has now entered clinical trials (phase II/III trials) for acute leukaemia therapy. ${ }^{96}$ Comparative in vitro cytotoxicity experiments demonstrated that the targeted camptothecin loaded-pegylated dendrimers had higher anti-proliferation activity towards nucleolin-positive HT29 and C26 colorectal cancer cells than the nucleolin-negative $\mathrm{CHO}$ cell line. The same system was tested in vivo on C26 tumor-bearing mice with promising results.

Another anti-cancer drug currently used in clinical settings is irinotecan. ${ }^{97}$ However, its utility is limited by its narrow therapeutic index. Theoretically, a possibility to improve the therapeutic index consists of increasing drug exposure in the diseased tissue, without accumulation in the healthy tissues. Dendrimer nanotechnology offers this possibility, as shown by England et $a l .{ }^{97}$ They modified generation 5 L-lysine dendrimers with polyoxazoline as a drug delivery vehicle for improving the therapeutic index of $\mathrm{SN}-38$, the active metabolite of irinotecan (Fig. 4D). This extensive study comprised different linker technologies to obtain diverse pharmacokinetic profiles of drug release. Three conjugates with plasma release half-lives of $2.5 \mathrm{~h}, 21 \mathrm{~h}$, and $72 \mathrm{~h}$ were tested for efficacy and toxicity using a mouse SW620 xenograft model. The linker with a plasma release half-life of $21 \mathrm{~h}$ achieved sustained SN-38 exposure in blood, above the target concentration. Overall, these extensive studies allowed the identification of a linker, a dose and a dosing regimen for SN-38 conjugated to polyoxazoline-modified dendrimer that maximized efficacy and minimized adverse side effects. ${ }^{97}$

Narmani et al. ${ }^{98}$ studied the anti-cancer efficacy of oxaliplatin (OX) using a nanocarrier system with enhanced targeting efficacy towards folic acid receptor (FAR) expressing CRC cells in vitro. ${ }^{98}$ This system consisted of PAMAM dendrimers G4 imprinted with polyethylene glycol (PEG) and folic acid. Polyethylene glycation of polymeric NPs is frequently applied to increase stability, and thus the half-life, as well as the non-immunogenic and non-antigenic properties. ${ }^{99,100}$ The PEG-PAMAM nano-complex containing OX was shown to have a superior cellular uptake in the SW480 cell line. The cell viability tests clearly demonstrated the cancer cell growth inhibition effects of PEG-PAMAM-FA-OX. These fundamental scientific advances (see Table 2), coupled with practical methods to covalently conjugate a wide range of bioactive molecules to the surface of a dendrimer or encapsulate

Table 2 Summary of different strategies using dendrimer NPs for CRC therapy

\begin{tabular}{lll}
\hline Type of dendrimer & Target & Anti-cancer drug \\
\hline PAMAM G4 & N/A & Capecitabine \\
Gold NPs inside PAMAM & MUC-1 aptamer & Curcumin \\
Pegylated PAMAM & AS1411 anti-nucleolin aptamers & Camptothecin \\
L-Lysine dendrimer G5 modified with polyoxazoline & N/A & SN-38 (active metabolite of Irinotecan) \\
PAMAM G4 & Folic acid & Oxaliplatin \\
PAMAM G5 & 8 trans-cyclooctene & N/A
\end{tabular}


them as guest molecules within void spaces, provide a highly versatile and potentially extremely powerful technological platform to fight colorectal cancer.

In another innovative approach, an in vivo pretargeting using radionuclides (that are usually incompatible with antibody-based vectors $\left(e . g .,{ }^{18} \mathrm{~F},{ }^{68} \mathrm{Ga},{ }^{11} \mathrm{C}\right.$, and $\left.{ }^{64} \mathrm{Cu}\right)$ ) helps imaging after the administration of the radiotracer and intensely decreases the dose of radiation doses to healthy tissues compared to the traditional protocol. ${ }^{101}$ For this, athymic nude mice bearing subcutaneous SW1222 human colorectal cancer xenografts were used. Indeed, pretargeted PET and biodistribution experiments in a murine model of colorectal carcinoma revealed that sshuA33-DEN-TCO (TCO-bearing dendrimers to huA33) produced dramatically improved tumoral activity concentrations compared to an analogous, dendrimer-lacking immunoconjugate (sshuA33PEG12-TCO-nondendrimeric control immunoconjugate). Surprisingly, the attachment of the G 0.5 dendrimeric structures did not hinder the in vivo performance of the immunoconjugate, suggesting that this bifunctional scaffold may have applications past pretargeting. ${ }^{101}$

\section{Conclusions}

The inefficacy of conventional chemotherapeutic methods has led to the development of new strategies, which can be used to improve the efficiency of anti-cancer drug delivery into tumors while minimizing distribution and toxicity in healthy tissues as well as novel imaging tools. These novel strategies based on the use of NPs loaded with drugs offer unprecedented opportunities both at the preclinical and clinical levels. However, some challenges still remain, such as improving the localization, biodistribution, biocompatibility, and efficacy of these nano-drug systems in vivo, to meet the requirements of precision cancer diagnosis and therapy. In the current scenario, among the new nanotechnology platforms, dendrimer-based chemotherapeutics have emerged as one of the most promising nanotools over the available conventional chemotherapies for the treatment of a variety of tumors. However, their application in colorectal cancer is still in its infancy.

An ideal therapeutic must have the ability to target cancer cells, image the extent of the tumor and sense its signatures, deliver a therapeutic, and monitor cells for their response. Although we are not there yet, this is the goal for nanotherapeutics.

Nanotechnology, and especially dendrimer nanoparticles with all of the above unique features, will support medical products to develop beyond a single mode of action into multifunctional platforms performing several functions such as nanotheranostics. Researchers around the world are enthusiastically incorporating nanotechnology in CRC treatment.

\section{Conflicts of interest}

There are no conflicts to declare.

\section{References}

1 L. Kotelevets, E. Chastre, D. Desmaële and P. Couvreur, Nanotechnologies for the treatment of colon cancer: From old drugs to new hope, Int. J. Pharm., 2016, 514, 24-40.

2 M. A. Gatoo, S. Naseem, M. Y. Arfat, A. M. Dar, K. Qasim and S. Zubair, Physicochemical properties of nanomaterials: implication in associated toxic manifestations, BioMed Res. Int., 2014, 2014, 498420.

3 P. Tekchandani, B. D. Kurmi and S. R. Paliwal, Nanomedicine to Deal With Cancer Cell Biology in Multi-Drug Resistance, Mini-Rev. Med. Chem., 2017, 17, 1793-1810.

4 M. Bar-Zeev, Y. D. Livney and Y. G. Assaraf, Targeted nanomedicine for cancer therapeutics: Towards precision medicine overcoming drug resistance, Drug Resist. Updates, 2017, 31, 15-30.

5 H. Maeda, H. Nakamura and J. Fang, The EPR effect for macromolecular drug delivery to solid tumors: Improvement of tumor uptake, lowering of systemic toxicity, and distinct tumor imaging in vivo, Adv. Drug Delivery Rev., 2013, 65, 71-79.

6 C. G. Da Silva, G. J. Peters, F. Ossendorp and L. J. Cruz, The potential of multi-compound nanoparticles to bypass drug resistance in cancer, Cancer Chemother. Pharmacol., 2017, 80, 881-894.

7 B. Zhitomirsky and Y. G. Assaraf, Lysosomes as mediators of drug resistance in cancer, Drug Resist. Updates, 2016, 24, 23-33.

8 M. R. Villegas, A. Baeza, A. Noureddine, P. N. Durfee, K. S. Butler and J. O. Agola, et al., Multifunctional Protocells for Enhanced Penetration in 3D Extracellular Tumoral Matrices, Chem. Mater., 2018, 30, 112-120.

9 E. Van Cutsem, C. H. Kohne, E. Hitre, J. Zaluski, C. R. Chang Chien and A. Makhson, et al., Cetuximab and chemotherapy as initial treatment for metastatic colorectal cancer, N. Engl. J. Med., 2009, 360, 1408-1417.

10 E. Kopansky, Y. Shamay and A. David, Peptide-directed HPMA copolymer-doxorubicin conjugates as targeted therapeutics for colorectal cancer, J. Drug Targeting, 2011, 19, 933-943.

11 K. J. Landmark, S. Dimaggio, J. Ward, C. Kelly, S. Vogt and S. Hong, et al., Synthesis, characterization, and in vitro testing of superparamagnetic iron oxide nanoparticles targeted using folic Acid-conjugated dendrimers, ACS Nano, 2008, 2, 773-783.

12 M. Alibolandi, F. Hoseini, M. Mohammadi, P. Ramezani, E. Einafshar and S. M. Taghdisi, et al., Curcumin-entrapped MUC-1 aptamer targeted dendrimer-gold hybrid nanostructure as a theranostic system for colon adenocarcinoma, Int. J. Pharm., 2018, 549, 67-75.

13 Z. Li, S. Tan, S. Li, Q. Shen and K. Wang, Cancer drug delivery in the nano era: An overview and perspectives (Review), Oncol. Rep., 2017, 38, 611-624.

14 E. Pavitra, B. Dariya, G. Srivani, S.-M. Kang, A. Alam and P.-R. Sudhir, et al., Engineered nanoparticles for imaging and drug delivery in colorectal cancer, Semin. Cancer Biol., 2019. 
15 H. Belhadj-Tahar, A. Chen, Y. Jia, S. Wu, N. Sadeg and $\mathrm{H}$. Zhao, et al., In situ anti-cancer agent derived from [188re]rhenium nitro-imidazole ligand loaded poly-Llysine dendrimer administrated by direct CT guided stereotactic intrahepatic injection, J. Clin. Oncol., 2018, 36, e15569.

16 H. Chang, H. Wang, N. Shao, M. Wang, X. Wang and Y. Cheng, Surface-engineered dendrimers with a diaminododecane core achieve efficient gene transfection and low cytotoxicity, Bioconjugate Chem., 2014, 25, 342-350.

17 R. Shukla, A. Singh, V. Pardhi, K. Kashyap, S. K. Dubey and R. Dandela, et al., Dendrimer-Based Nanoparticulate Delivery System for Cancer Therapy, in Polymeric Nanoparticles as a Promising Tool for Anti-cancer Therapeutics, ed. P. Kesharwani, K. M. Paknikar and V. Gajbhiye, Academic Press, 2019, ch. 11, pp. 233-255.

18 J. Elgqvist, Nanoparticles as Theranostic Vehicles in Experimental and Clinical Applications-Focus on Prostate and Breast Cancer, Int. J. Mol. Sci., 2017, 18, E1102.

19 J. Singh, K. Jain, N. K. Mehra and N. K. Jain, Dendrimers in anticancer drug delivery: mechanism of interaction of drug and dendrimers, Artif. Cells, Nanomed., Biotechnol., 2016, 44, 1626-1634.

20 M. Stanczyk, A. Dziki and Z. Morawiec, Dendrimers in therapy for breast and colorectal cancer, Curr. Med. Chem., 2012, 19, 4896-4902.

21 N. Amreddy, R. A. Ahmed, A. Munshi and R. Ramesh, Tumor-Targeted Dendrimer Nanoparticles for Combinatorial Delivery of siRNA and Chemotherapy for Cancer Treatment, Methods Mol. Biol., 2020, 2059, 167-189.

22 R. V. D. Araújo, S. D. S. Santos, E. Igne Ferreira and J. Giarolla, New Advances in General Biomedical Applications of PAMAM Dendrimers, Molecules, 2018, 23, 2849.

23 S. Lee, S. J. Son, S. J. Song, T. H. Ha and J. S. Choi, Polyamidoamine (PAMAM) Dendrimers Modified with Cathepsin-B Cleavable Oligopeptides for Enhanced Gene Delivery, Polymers, 2017, 9, 224.

24 D. A. Tomalia and J. M. J. Fréchet, Discovery of dendrimers and dendritic polymers: A brief historical perspective, J. Polym. Sci., Part A: Polym. Chem., 2002, 40, 2719-2728.

25 J. M. Oliveira, A. J. Salgado, N. Sousa, J. F. Mano and R. L. Reis, Dendrimers and derivatives as a potential therapeutic tool in regenerative medicine strategies-A review, Prog. Polym. Sci., 2010, 35, 1163-1194.

26 B. Srinageshwar, S. Peruzzaro, M. Andrews, K. Johnson, A. Hietpas and B. Clark, et al., PAMAM Dendrimers Cross the Blood-Brain Barrier When Administered through the Carotid Artery in C57BL/6J Mice, Int. J. Mol. Sci., 2017, 18, 628.

27 E. Hill, R. Shukla, S. S. Park and J. R. Baker, Jr., Synthetic PAMAM-RGD conjugates target and bind to odontoblastlike MDPC 23 cells and the predentin in tooth organ cultures, Bioconjugate Chem., 2007, 18, 1756-1762.

28 M. Salimi, S. Sarkar, S. Fathi, A. M. Alizadeh, R. Saber and F. Moradi, et al., Biodistribution, pharmacokinetics, and toxicity of dendrimer-coated iron oxide nanoparticles in BALB/c mice, Int. J. Nanomed., 2018, 13, 1483-1493.
29 T. P. Thomas, A. K. Patri, A. Myc, M. T. Myaing, J. Y. Ye and T. B. Norris, et al., In vitro targeting of synthesized antibody-conjugated dendrimer nanoparticles, Biomacromolecules, 2004, 5, 2269-2274.

30 L. M. Kaminskas, B. J. Boyd and C. J. Porter, Dendrimer pharmacokinetics: the effect of size, structure and surface characteristics on ADME properties, Nanomedicine, 2011, 6, 1063-1084.

31 W. G. Lesniak, M. K. Mishra, A. Jyoti, B. Balakrishnan, F. Zhang and E. Nance, et al., Biodistribution of Fluorescently Labeled PAMAM Dendrimers in Neonatal Rabbits: Effect of Neuroinflammation, Mol. Pharmaceutics, 2013, 10, 4560-4571.

32 H. Kobayashi, S. Kawamoto, S. K. Jo, N. Sato, T. Saga and A. Hiraga, et al., Renal tubular damage detected by dynamic micro-MRI with a dendrimer-based magnetic resonance contrast agent, Kidney Int., 2002, 61, 1980-1985.

33 A. K. Sharma, A. Gothwal, P. Kesharwani, H. Alsaab, A. K. Iyer and U. Gupta, Dendrimer nanoarchitectures for cancer diagnosis and anticancer drug delivery, Drug Discovery Today, 2017, 22, 314-326.

34 C. J. Hawker and J. M. J. Frechet, Preparation of polymers with controlled molecular architecture. A new convergent approach to dendritic macromolecules, J. Am. Chem. Soc., 1990, 112, 7638-7647.

35 E. Abbasi, S. F. Aval, A. Akbarzadeh, M. Milani, H. T. Nasrabadi and S. W. Joo, et al., Dendrimers: synthesis, applications, and properties, Nanoscale Res. Lett., 2014, 9, 247.

36 D. A. Tomalia, Birth of a new macromolecular architecture: dendrimers as quantized building blocks for nanoscale synthetic polymer chemistry, Prog. Polym. Sci., 2005, 30, 294-324.

37 J. M. Oliveira, N. Kotobuki, A. P. Marques, R. P. Pirraco, J. Benesch and M. Hirose, et al., Surface Engineered Carboxymethylchitosan/Poly(amidoamine) Dendrimer Nanoparticles for Intracellular Targeting, Adv. Funct. Mater., 2008, 18, 1840-1853.

38 L. Palmerston Mendes, J. Pan and V. P. Torchilin, Dendrimers as Nanocarriers for Nucleic Acid and Drug Delivery in Cancer Therapy, Molecules, 2017, 22, 1401.

39 Z. Lyu, L. Ding, A. Y. T. Huang, C. L. Kao and L. Peng, Poly(amidoamine) dendrimers: covalent and supramolecular synthesis, Mater. Today Chem., 2019, 13, 34-48.

40 E. Pedziwiatr-Werbicka, K. Milowska, V. Dzmitruk, M. Ionov, D. Shcharbin and M. Bryszewska, Dendrimers and hyperbranched structures for biomedical applications, Eur. Polym. J., 2019, 119, 61-73.

41 S. K. Shukla, P. P. Govender and A. Tiwari, Polymeric Micellar Structures for Biosensor Technology, in Advances in Biomembranes and Lipid Self-Assembly, ed. A. Iglič, C. V. Kulkarni, M. Rappolt, Academic Press, 2016, ch. 6, pp. 143-161.

42 D. Kaur, K. Jain, N. K. Mehra, P. Kesharwani and N. K. Jain, A review on comparative study of PPI and PAMAM dendrimers, J. Nanopart. Res., 2016, 18, 146. 
43 K. Madaan, S. Kumar, N. Poonia, V. Lather and D. Pandita, Dendrimers in drug delivery and targeting: Drug-dendrimer interactions and toxicity issues, J. Pharm. BioAllied Sci., 2014, 6, 139-150.

44 P. Kesharwani, K. Jain and N. K. Jain, Dendrimer as nanocarrier for drug delivery, Prog. Polym. Sci., 2014, 39, 268-307.

45 L. Uram, A. Filipowicz, M. Misiorek, N. Pienkowska, J. Markowicz and E. Walajtys-Rode, et al., Biotinylated PAMAM G3 dendrimer conjugated with celecoxib and/or Fmoc-L-Leucine and its cytotoxicity for normal and cancer human cell lines, Eur. J. Pharm. Sci., 2018, 124, 1-9.

46 H. Cong, L. Zhou, Q. Meng, Y. Zhang, B. Yu and Y. Shen, et al., Preparation and evaluation of PAMAM dendrimerbased polymer gels physically cross-linked by hydrogen bonding, Biomater. Sci., 2019, 7, 3918-3925.

47 E. Abbasi, S. F. Aval, A. Akbarzadeh, M. Milani, H. T. Nasrabadi and S. W. Joo, et al., Dendrimers: synthesis, applications, and properties, Nanoscale Res. Lett., 2014, 9, 247.

48 D. A. Tomalia, H. Baker, J. Dewald, M. Hall, G. Kallos and S. Martin, et al., Dendritic macromolecules: synthesis of starburst dendrimers, Macromolecules, 1986, 19, 2466-2468.

49 J. Abraham, B. A. Aderibigbe, H. Afshar, V. Aina, N. Alam and S. Banerjee, et al., List of Contributors, in Nanoarchitectonics for Smart Delivery and Drug Targeting, ed. A. M. Holban and A. M. Grumezescu, William Andrew Publishing, 2016, pp. xix-xxvi.

50 A. S. Chauhan and M. Kaul, Engineering of "critical nanoscale design parameters" (CNDPs) in PAMAM dendrimer nanoparticles for drug delivery applications, J. Nanopart. Res., 2018, 20, 226.

51 X. Cai, J. Hu, J. Xiao and Y. Cheng, Dendrimer and cancer: a patent review (2006-present), Expert Opin. Ther. Pat., 2013, 23, 515-529.

52 M. Ghaffari, G. Dehghan, F. Abedi-Gaballu, S. Kashanian, B. Baradaran and J. Ezzati Nazhad Dolatabadi, et al., Surface functionalized dendrimers as controlled-release delivery nanosystems for tumor targeting, Eur. J. Pharm. Sci., 2018, 122, 311-330.

53 S. Somani, P. Laskar, N. Altwaijry, P. Kewcharoenvong, C. Irving and G. Robb, et al., PEGylation of polypropylenimine dendrimers: effects on cytotoxicity, DNA condensation, gene delivery and expression in cancer cells, Sci. Rep., 2018, 8, 9410.

54 I. J. Majoros, T. P. Thomas, C. B. Mehta and J. R. Baker, Jr., Poly(amidoamine) dendrimer-based multifunctional engineered nanodevice for cancer therapy, J. Med. Chem., 2005, 48, 5892-5899.

55 R. S. Navath, A. R. Menjoge, B. Wang, R. Romero, S. Kannan and R. M. Kannan, Amino Acid-Functionalized Dendrimers with Heterobifunctional Chemoselective Peripheral Groups for Drug Delivery Applications, Biomacromolecules, 2010, 11, 1544-1563.

56 P. P. Pednekar, K. R. Jadhav and V. J. Kadam, Aptamerdendrimer bioconjugate: a nanotool for therapeutics, diagnosis, and imaging, Expert Opin. Drug Delivery, 2012, 9, 1273-1288.

57 J. B. Otis, H. Zong, A. Kotylar, A. Yin, S. Bhattacharjee and H. Wang, et al., Dendrimer antibody conjugate to target and image HER-2 overexpressing cancer cells, Oncotarget, 2016, 7, 36002-36013.

58 L. Uram, M. Szuster, M. Misiorek, A. Filipowicz, S. Wolowiec and E. Walajtys-Rode, The effect of G3 PAMAM dendrimer conjugated with B-group vitamins on cell morphology, motility and ATP level in normal and cancer cells, Eur. J. Pharm. Sci., 2017, 102, 275-283.

59 M. R. Carvalho, C. R. Carvalho, F. R. Maia, D. Caballero, S. C. Kundu and R. L. Reis, et al., Peptide-Modified Dendrimer Nanoparticles for Targeted Therapy of Colorectal Cancer, Adv. Ther., 2019, 2, 1900132.

60 N. Zhao, Y. Qin, H. Liu and Z. Cheng, Tumor-Targeting Peptides: Ligands for Molecular Imaging and Therapy, Anti-Cancer Agents Med. Chem., 2018, 18, 74-86.

61 M. Zhang, J. Zhu, Y. Zheng, R. Guo, S. Wang and S. Mignani, et al., Doxorubicin-Conjugated PAMAM Dendrimers for $\mathrm{pH}$-Responsive Drug Release and Folic Acid-Targeted Cancer Therapy, Pharmaceutics, 2018, 10, 162.

62 W. Wu, W. Driessen and X. Jiang, Oligo(ethylene glycol)Based Thermosensitive Dendrimers and Their Tumor Accumulation and Penetration, J. Am. Chem. Soc., 2014, 136, 3145-3155.

63 S.-Y. Wu, H.-Y. Chou, C.-H. Yuh, S. L. Mekuria, Y.-C. Kao and H.-C. Tsai, Radiation-Sensitive Dendrimer-Based Drug Delivery System, Adv. Sci., 2017, 5, 1700339.

64 P. Laskar, S. Somani, S. J. Campbell, M. Mullin, P. Keating and R. J. Tate, et al., Camptothecin-based dendrimersomes for gene delivery and redox-responsive drug delivery to cancer cells, Nanoscale, 2019, 11, 20058-20071.

65 R. V. Araujo, S. D. S. Santos, E. Igne Ferreira and J. Giarolla, New Advances in General Biomedical Applications of PAMAM Dendrimers, Molecules, 2018, 23, 2849.

66 M. R. Carvalho, D. Barata, L. M. Teixeira, S. Giselbrecht, R. L. Reis and J. M. Oliveira, et al., Colorectal tumor-on-achip system: A 3D tool for precision onco-nanomedicine, Sci. Adv., 2019, 5, eaaw1317.

67 J. M. Oliveira, R. A. Sousa, N. Kotobuki, M. Tadokoro, M. Hirose and J. F. Mano, et al., The osteogenic differentiation of rat bone marrow stromal cells cultured with dexamethasone-loaded carboxymethylchitosan/poly(amidoamine) dendrimer nanoparticles, Biomaterials, 2009, 30, 804-813.

68 L. Zhao, X. Shi and J. Zhao, Dendrimer-based contrast agents for PET imaging, Drug Delivery, 2017, 24, 81-93.

69 Y. Huang, D. Coman, F. Hyder and M. M. Ali, DendrimerBased Responsive MRI Contrast Agents (G1-G4) for Biosensor Imaging of Redundant Deviation in Shifts (BIRDS), Bioconjugate Chem., 2015, 26, 2315-2323.

70 A.-M. Caminade, S. Fruchon, C.-O. Turrin, M. Poupot, A. Ouali and A. Maraval, et al., The key role of the scaffold on the efficiency of dendrimer nanodrugs, Nat. Commun., 2015, 6, 7722 . 
71 J. R. Baker, Jr., Dendrimer-based nanoparticles for cancer therapy, Hematology, 2009, 708-719.

72 V. Saluja, A. Mankoo, G. K. Saraogi, M. M. Tambuwala and V. Mishra, Smart dendrimers: Synergizing the targeting of anticancer bioactives, J. Drug Delivery Sci. Technol., 2019, 52, 15-26.

73 A. Kesavan, S. B. Pakala, S. K. Rayala and G. Venkatraman, Effective Strategies and Applications of Dendrimers in the Treatment of Ovarian Cancer, Curr. Pharm. Des., 2017, 23, 3099-3104.

74 S. Mura, J. Nicolas and P. Couvreur, Stimuli-responsive nanocarriers for drug delivery, Nat. Mater., 2013, 12, 991.

75 S. Gai, G. Yang, P. Yang, F. He, J. Lin and D. Jin, et al., Recent advances in functional nanomaterials for lighttriggered cancer therapy, Nano Today, 2018, 19, 146-187.

76 S. K. Choi, T. Thomas, M. H. Li, A. Kotlyar, A. Desai and J. R. Baker, Jr., Light-controlled release of caged doxorubicin from folate receptor-targeting PAMAM dendrimer nanoconjugate, Chem. Commun., 2010, 46, 2632-2634.

77 S. Nigam and D. Bahadur, Dendrimer-conjugated iron oxide nanoparticles as stimuli-responsive drug carriers for thermally-activated chemotherapy of cancer, Colloids Surf., B, 2017, 155, 182-192.

78 N. Amreddy, A. Babu, J. Panneerselvam, A. Srivastava, R. Muralidharan and A. Chen, et al., Chemo-biologic combinatorial drug delivery using folate receptor-targeted dendrimer nanoparticles for lung cancer treatment, Nanomedicine, 2018, 14, 373-384.

79 C. Chittasupho, S. Anuchapreeda and N. Sarisuta, CXCR4 targeted dendrimer for anti-cancer drug delivery and breast cancer cell migration inhibition, Eur. J. Pharm. Biopharm., 2017, 119, 310-321.

80 S. Yousef, H. O. Alsaab, S. Sau and A. K. Iyer, Development of asialoglycoprotein receptor directed nanoparticles for selective delivery of curcumin derivative to hepatocellular carcinoma, Heliyon, 2018, 4, e01071.

81 T. J. Lee, J. Y. Yoo, D. Shu, H. Li, J. Zhang and J. G. Yu, et al., RNA Nanoparticle-Based Targeted Therapy for Glioblastoma through Inhibition of Oncogenic miR-21, Mol. Ther., 2017, 25, 1544-1555.

82 V. Mishra and P. Kesharwani, Dendrimer technologies for brain tumor, Drug Discovery Today, 2016, 21, 766-778.

83 S. Liu, Y. Guo, R. Huang, J. Li, S. Huang and Y. Kuang, et al., Gene and doxorubicin co-delivery system for targeting therapy of glioma, Biomaterials, 2012, 33, 4907-4916.

84 X. X. Wu, Y. Kakehi, Y. Mizutani, T. Kamoto, H. Kinoshita and Y. Isogawa, et al., Doxorubicin enhances TRAILinduced apoptosis in prostate cancer, Int. J. Oncol., 2002, 20, 949-954.

85 Z. Zhou and Z.-R. Lu, Gadolinium-based contrast agents for magnetic resonance cancer imaging, Wiley Interdiscip. Rev.: Nanomed. Nanobiotechnol., 2013, 5, 1-18.

86 V. S. Talanov, C. A. Regino, H. Kobayashi, M. Bernardo, P. L. Choyke and M. W. Brechbiel, Dendrimer-based nanoprobe for dual modality magnetic resonance and fluorescence imaging, Nano Lett., 2006, 6, 1459-1463.
87 M. Bohorquez, R. Sahasrabudhe, A. Criollo, M. C. SanabriaSalas, A. Vélez and J. M. Castro, et al., Clinical manifestations of colorectal cancer patients from a large multicenter study in Colombia, Medicine, 2016, 95, e4883.

88 B. A. Cisterna, N. Kamaly, W. I. Choi, A. Tavakkoli, O. C. Farokhzad and C. Vilos, Targeted nanoparticles for colorectal cancer, Nanomedicine, 2016, 11, 2443-2456.

89 M. Fleming, S. Ravula, S. F. Tatishchev and H. L. Wang, Colorectal carcinoma: Pathologic aspects, J. Gastrointest. Oncol., 2012, 3, 153-173.

90 M. Ghosn, H. R. Kourie and S. Tabchi, Gastrointestinal cancers in the era of theranostics: Updates and future perspectives, World J. Gastroenterol., 2015, 21, 8473-8477.

91 W. Yan, M. Tao, B. Jiang, M. Yao, Y. Jun and W. Dai, et al., Overcoming Drug Resistance in Colon Cancer by AptamerMediated Targeted Co-Delivery of Drug and siRNA Using Grapefruit-Derived Nanovectors, Cell. Physiol. Biochem., 2018, 50, 79-91.

92 F. Nabavizadeh, H. Fanaei, A. Imani, J. Vahedian, F. Asadi Amoli and J. Ghorbi, et al., Evaluation of Nanocarrier Targeted Drug Delivery of Capecitabine-PAMAM Dendrimer Complex in a Mice Colorectal Cancer Model, Acta Med. Iran., 2016, 54, 485-493.

93 J. Xie, J. Wang, H. Chen, W. Shen, P. J. Sinko and H. Dong, et al., Multivalent Conjugation of Antibody to Dendrimers for the Enhanced Capture and Regulation on Colon Cancer Cells, Sci. Rep., 2015, 5, 9445.

94 A. Gulbake, A. Jain, A. Jain, A. Jain and S. K. Jain, Insight to drug delivery aspects for colorectal cancer, World J. Gastroenterol., 2016, 22, 582-599.

95 M. Alibolandi, S. M. Taghdisi, P. Ramezani, F. Hosseini Shamili, S. A. Farzad and K. Abnous, et al., Smart AS1411aptamer conjugated pegylated PAMAM dendrimer for the superior delivery of camptothecin to colon adenocarcinoma in vitro and in vivo, Int. J. Pharm., 2017, 519, 352-364.

96 X. Fan, L. Sun, Y. Wu, L. Zhang and Z. Yang, Bioactivity of 2'-deoxyinosine-incorporated aptamer AS1411, Sci. Rep., 2016, 6, 25799.

97 R. M. England, J. I. Hare, J. Barnes, J. Wilson, A. Smith and N. Strittmatter, et al., Tumour regression and improved gastrointestinal tolerability from controlled release of $\mathrm{SN}-38$ from novel polyoxazoline-modified dendrimers, J. Controlled Release, 2017, 247, 73-85.

98 A. Narmani, M. Kamali, B. Amini, A. Salimi and Y. Panahi, Targeting delivery of oxaliplatin with smart PEG-modified PAMAM G4 to colorectal cell line: In vitro studies, Process Biochem., 2018, 69, 178-187.

99 R. I. Castro, O. Forero-Doria and L. Guzman, Perspectives of Dendrimer-based Nanoparticles in Cancer Therapy, An. Acad. Bras. Cienc., 2018, 90, 2331-2346.

100 M. E. Fox, S. Guillaudeu, J. M. J. Fréchet, K. Jerger, N. Macaraeg and F. C. Szoka, Synthesis and in vivo antitumor efficacy of PEGylated poly(l-lysine) dendrimer-camptothecin conjugates, Mol. Pharmaceutics, 2009, 6, 1562-1572.

101 B. E. Cook, R. Membreno and B. M. Zeglis, Dendrimer Scaffold for the Amplification of In Vivo Pretargeting Ligations, Bioconjugate Chem., 2018, 29, 2734-2740. 